\title{
Quasi-spherical gravitational collapse and the role of initial data, anisotropy and inhomogeneity
}

\author{
Subenoy Chakraborty* and Ujjal Debnath \\ Department of Mathematics, Jadavpur University, Calcutta-32, India.
}

(Dated: January 8, 2019)

\begin{abstract}
In this paper, the role of anisotropy and inhomogeneity has been studied in quasi-spherical gravitational collapse. Also the role of initial data has been investigated in characterizing the final state of collapse. Finally, a linear transformation on the initial data set has been presented and its impact has been discussed.
\end{abstract}

PACS numbers:

\section{INTRODUCTION}

Gravitational collapse is an important and challenging issue in Einstein gravity for the last two decades or more, particularly after the formation of famous singularity theorems [1] and Cosmic Censorship Conjecture (CCC) [2]. Moreover, the final outcome of gravitational collapse [3] in the background of general relativity is interesting both from the point of view of black hole physics as well as its astrophysical implications. In fact, an extensive study of gravitational collapse [4 - 9] has been carried out of Tolman-Bondi-Lemaitre (TBL) spherically symmetric space-times containing irrotational dust to support or disprove the CCC. A general conclusion from these studies is that a central curvature singularity forms but its local or global visibility depends on the initial data. Recently, mena et al [10] have introduced a linear transformation on the initial data set which keeps $\rho$ and $\sigma$ to be invariant at any time instant except for the initial ones and have discussed the consequences for the final fate of collapse.

On the otherhand, there is very little progress in studying non-spherical collapse due to the ambiguity of horizon formation and the influence of gravitational radiation. However, in recent past, an extensive study of irrotational dust collapse has been done in quasi-spherical Szekeres space-time [11-13]. In this paper, we have extended the work of Mena et al to quasi-spherical Szekeres model and have investigated the effect of inhomogeneity and anisotropy on the final nature of singularity. The paper is organized as follows: a brief outline of the quasi-spherical dust collapse has been presented in section II. Section III deals with properties of inhomogeneity and anisotropy both initially and at any time instant and then linear transformation on the initial data set have been discussed. The paper ends with conclusion in section IV.

\section{QUASI-SPHERICAL DUST COLLAPSE}

The inhomogeneous quasi-spherical dust collapse in $n$-dimension is represented by Szekeres' space-time with line element

$$
d s^{2}=-d t^{2}+e^{2 \alpha} d r^{2}+e^{2 \beta} \sum_{i=1}^{n-2} d x_{i}^{2}
$$

\footnotetext{
*Electronic address: subenoyc@yahoo.co.in

†Electronic address: ujjaldebnath@yahoo.com
} 
where the metric coefficients $\alpha$ and $\beta$ are functions of all the $n$ space-time co-ordinates and have explicit form $[12,14]$

$$
e^{\alpha}=\frac{R^{\prime}+R \nu^{\prime}}{\sqrt{1+f(r)}}, \quad e^{\beta}=R(t, r) e^{\nu\left(r, x_{1}, \ldots, x_{n}\right)}
$$

The evolution equation for $R$ is

$$
\dot{R}^{2}=f(r)+\frac{F(r)}{R^{n-3}}
$$

and $\nu$ has the explicit form

$$
e^{-\nu}=A(r) \sum_{i=1}^{n-2} x_{i}^{2}+\sum_{i=1}^{n-2} B_{i}(r) x_{i}+C(r)
$$

where $F(r)(>0)$ and $f(r)(>-1)$ are arbitrary functions of $r$ and $A(r), B_{i}(r)$ 's and $C(r)$ are arbitrary functions of $r$ alone with the restriction

$$
\sum_{i=1}^{n-2} B_{i}^{2}-4 A C=-1
$$

(dot and dash stands for partial differentiation with respect to $t$ and $r$ respectively).

The matter density and the anisotropy scalar are given by [12]

$$
\rho\left(t, r, x_{1}, \ldots, x_{n-2}\right)=\frac{(n-2)}{2}\left[\frac{F^{\prime}+(n-1) F \nu^{\prime}}{R^{n-2}\left(R^{\prime}+R \nu^{\prime}\right)}\right]
$$

and

$$
\sigma\left(t, r, x_{1}, \ldots, x_{n-2}\right)=\sqrt{\frac{n-2}{2(n-1)}}\left[\frac{R \dot{R}^{\prime}-\dot{R} R^{\prime}}{R\left(R^{\prime}+R \nu^{\prime}\right)}\right]
$$

Now integrating the evolution equation (3) we have

$$
t-t_{i}=\frac{2}{(n-1) \sqrt{F}}\left[r^{\frac{n-1}{2}}{ }_{2} F_{1}\left[\frac{1}{2}, a, a+1,-\frac{f r^{n-3}}{F}\right]-R^{\frac{n-1}{2}}{ }_{2} F_{1}\left[\frac{1}{2}, a, a+1,-\frac{f R^{n-3}}{F}\right]\right]
$$

where $R=r$ at the initial epoch $t=t_{i}$ and ${ }_{2} F_{1}$ is the usual hypergeometric function with $a=\frac{1}{2}+\frac{1}{n-3}$.

If $t=t_{s}(r)$ stands for time of collapse of the $r$-th shell i.e., $R\left(t_{s}(r), r\right)=0$ then we have

$$
t_{s}(r)-t_{i}=\frac{2}{(n-1) \sqrt{F}} r^{\frac{n-1}{2}}{ }_{2} F_{1}\left[\frac{1}{2}, a, a+1,-\frac{f r^{n-3}}{F}\right]
$$

Further, at the time of formation of trapped surfaces (i.e., $\left.t=t_{a h}(r)\right)$ we have $R^{n-3}=$ $F(r)$, so we get

$$
t_{a h}(r)-t_{i}=\frac{2 r^{\frac{n-1}{2}}}{(n-1) \sqrt{F}}{ }_{2} F_{1}\left[\frac{1}{2}, a, a+1,-\frac{f r^{n-3}}{F}\right]-\frac{2 F^{\frac{1}{n-3}}}{n-1}{ }_{2} F_{1}\left[\frac{1}{2}, a, a+1,-f\right]
$$


The time of formation of central singularity is given by

$$
t_{0}=t_{s}(0)=t_{i}+\lim _{r \rightarrow 0} \frac{2 r^{\frac{n-1}{2}}}{(n-1) \sqrt{F}}{ }_{2} F_{1}\left[\frac{1}{2}, a, a+1,-\frac{f r^{n-3}}{F}\right]
$$

For finite value of the above limit, we assume $F(r)$ and $f(r)$ to be in the following polynomial form near the central singularity $(r=0)$

$$
\begin{gathered}
F(r)=F_{0} r^{n-1}+F_{1} r^{n}+F_{2} r^{n+1}+\ldots \ldots \ldots, \\
f(r)=f_{0} r^{2}+f_{1} r^{3}+f_{2} r^{4}+\ldots \ldots \ldots
\end{gathered}
$$

Then equation (6) suggests that initial density profile $\rho_{i}\left(=\rho\left(t_{i}, r\right)\right)$ is smooth at the centre and we have

$$
\rho_{i}(r)=\rho_{0}+\rho_{1} r+\rho_{2} r^{2}+\ldots \ldots \ldots
$$

Now using the initial condition $R\left(t_{i}, r\right)=r$ the initial density and shear have the expressions

$$
\rho_{i}=\frac{(n-2)}{2} \frac{F^{\prime}+(n-1) F \nu^{\prime}}{r^{n-2}\left(1+r \nu^{\prime}\right)}
$$

and

$$
\sigma_{i}=\sqrt{\frac{n-2}{8(n-1)}} \frac{\left[\left\{r F^{\prime}-(n-1) F\right\}+r^{n-3}\left(r f^{\prime}-2 f\right)\right]}{r^{\frac{n-1}{2}}\left(F+f r^{n-3}\right)^{1 / 2}\left(1+r \nu^{\prime}\right)}
$$

Using the series expansions (12) and (13) in equation (14) and comparing terms of equal powers in $r$ we have

$$
\rho_{j}=\frac{(n+j-1)(n-2)}{2} F_{j}, \quad j=0,1,2, \ldots
$$

Further, the above series expansions result (after simplification) an explicit expression for the time difference between the formation of trapped surface and the occurrence of central singularity as (upto leading order in $r$ )

$$
\begin{aligned}
t_{a h}-t_{0}= & -\frac{2}{n-1} F_{0}^{\frac{1}{n-3}} r^{\frac{n-1}{n-3}}-\frac{r}{(n-1) \sqrt{F}}\left[\frac{F_{1}}{F_{0}}{ }_{2} F_{1}\left[\frac{1}{2}, a, a+1,-\frac{f_{0}}{F_{0}}\right]\right. \\
& \left.+\frac{(n-1)}{(3 n-7)} \frac{f_{0}}{F_{0}}\left(\frac{f_{1}}{f_{0}}-\frac{F_{1}}{F_{0}}\right){ }_{2} F_{1}\left[\frac{3}{2}, a+1, a+2,-\frac{f_{0}}{F_{0}}\right]\right]
\end{aligned}
$$

The necessary condition for existence of naked singularity is characterized by $t_{a h}(r) \geq t_{0}$. Hence the nature is completely specified by the initial data set $\mathcal{I}=\{F, f\}$. It is to be noted that if the coefficient of $r$ in the expression (17) vanishes then the end state of collapse also depends on the dimension of the space-time.

\section{A STUDY ON ANISOTROPY AND INHOMOGENEITY}

In this section we shall study some characteristic of anisotropy and inhomogeneity of the space-time, we shall state them in the form of proposition. 


\section{A. Some Results}

Proposition I: If $\sigma\left(t_{i}, r\right)=0=\dot{\sigma}\left(t_{i}, r\right)$ then the initial data set is homogeneous and the initial space-time is homogeneous and isotropic.

Proof: The expression for $\dot{\sigma}\left(t_{i}, r\right)$ is

$$
\begin{aligned}
\dot{\sigma}\left(t_{i}, r, x_{1}, \ldots, x_{n-2}\right) & =\sqrt{\frac{n-2}{2(n-1)}} \frac{(n-3)\left[(n-1) F-r F^{\prime}\right]}{2 r\left(1+r \nu^{\prime}\right)} \\
& -\frac{\sigma_{i}\left[\frac{F\left(5-n+2 r \nu^{\prime}\right)}{r^{n-3}}+f\left(1+r \nu^{\prime}\right)+r\left(f^{\prime}+\frac{F^{\prime}}{r^{n-3}}\right)\right]}{2 r\left(1+r \nu^{\prime}\right) \sqrt{\frac{F}{r^{n-3}}+f}}
\end{aligned}
$$

Hence $\dot{\sigma}=0=\sigma_{i}$ implies

$$
(n-1) F-r F^{\prime}=0
$$

i.e.,

$$
F=F_{0} r^{n-1}
$$

Also $\sigma_{i}=0$ results

$$
\frac{r F^{\prime}-(n-1) F}{r^{n-3}}+\left(r f^{\prime}-2 f\right)=0
$$

So using (19) we have

$$
r f^{\prime}-2 f=0 \text { i.e., } f=f_{0} r^{2} .
$$

Thus the initial data is homogeneous.

The expression for initial density contrast is

$$
\begin{aligned}
\left.\frac{\partial \rho}{\partial t}\right|_{t=t_{i}}= & \frac{(n-2)}{2}\left[\frac{F^{\prime \prime}}{r^{n-2}\left(1+r \nu^{\prime}\right)}-\frac{(n-2) F^{\prime}}{r^{n-1}\left(1+r \nu^{\prime}\right)^{2}}\right. \\
& \left.+\frac{\left\{-(n-1)(n-2) F \nu^{\prime}+r\left(r F^{\prime}-(n-1) F\right)\left((n-1) \nu^{\prime 2}-\nu^{\prime \prime}\right)\right\}}{r^{n-1}\left(1+r \nu^{\prime}\right)}\right]
\end{aligned}
$$

which vanishes identically for the choice of $F$ in equation (20). It is to be noted that this choice of $F$ simplifies the initial density to

$$
\rho_{i}=\frac{(n-1)(n-2)}{2} F_{0}
$$

which is homogeneous. Hence the initial space-time is also homogeneous and isotropic.

Proposition II: If $\sigma\left(t, r, x_{1}, \ldots, x_{n-2}\right)=0$ then (i) the initial data set is homogeneous, (ii) the space-time is homogeneous and isotropic, (iii) the end state of collapse will always be a black hole. 
Proof: From equation (7), $\sigma=0$ implies

$$
\frac{\dot{R}^{\prime}}{R^{\prime}}=\frac{\dot{R}}{R}
$$

which shows that $R=g(r) \mu(t)$. But at $t=t_{i}, R=r$, hence we have

$$
R(t, r)=r \mu(t)
$$

Now substituting in the evolution equation for $R$ (i.e., eq.(3)) we have

$$
\dot{\mu}^{2}=\frac{F_{0}}{\mu^{n-3}}+f_{0}
$$

with $F=F_{0} r^{n-1}, f=f_{0} r^{2}$ i.e., the initial data set is homogeneous.

So the expression for density simplifies to

$$
\rho(t)=\frac{(n-1)(n-2) F_{0}}{2 \mu^{n-1}}
$$

Hence the space-time is homogeneous and isotropic (by condition).

Further, using this expression for $R$ (i.e., eq.(24)) the time difference between the formation of trapped surfaces $\left(t_{a h}\right)$ and the occurrence of central singularity $\left(t_{0}\right)$ becomes

$$
t_{a h}-t_{0}=-\frac{2}{(n-1)} F_{0}^{\frac{1}{n-3}} r^{\frac{n-1}{n-3}}{ }_{2} F_{1}\left[\frac{1}{2}, a, a+1,-f_{0} r^{2}\right]
$$

So always we have $t_{a h}<t_{0}$ and the end state of collapse will be a black hole. It is to be noted that similar results holds also for spherical collapse [14].

Proposition III: If $\rho_{i}^{\prime}=0$ then the initial data set is homogeneous as well as the initial space-time is homogeneous and isotropic.

Proof: The expression for $\rho_{i}^{\prime}$ can be simplified to

$$
\begin{aligned}
& \rho_{i}^{\prime}=\frac{(n-2)}{2 r^{n-1}\left(1+r \nu^{\prime}\right)^{2}}\left[\left(1+r \nu^{\prime}\right)\left\{r F^{\prime \prime}-(n-2) F^{\prime}\right\}\right. \\
& \left.\quad+\left\{r F^{\prime}-(n-1) F\right\}\left\{(n-2)+(n-1) r \nu^{\prime 2}-r \nu^{\prime \prime}\right\}\right]
\end{aligned}
$$

which shows that for $\rho_{i}^{\prime}=0$ we can choose $F=F_{0} r^{n-1}$. Also for regularity of initial shear at $r=0$ demands $f=f_{0} r^{2}$. Hence the initial data set is homogeneous. For this choice of $F$ and $f, \rho_{i}$ becomes constant and $\sigma_{i}=0$. Therefore, the initial space-time is homogeneous and isotropic.

Proposition IV: If the density contrast vanishes at any time instant (i.e., $\left.\rho^{\prime}\left(t, r, x_{1}, \ldots, x_{n-2}\right)=0\right)$, starting from regular initial data, then the space-time will be homogeneous and isotropic and final state of collapse will be a black hole.

Proof: Using the condition for regular initial data (i.e., $F=F_{0} r^{n-1}, f=f_{0} r^{2}$ ) the expression for energy density simplifies to

$$
\rho\left(t, r, x_{1}, \ldots, x_{n-2}\right)=\frac{(n-1)(n-2)}{2} \frac{r^{n-2}\left(1+r \nu^{\prime}\right) F_{0}}{R^{n-2}\left(R^{\prime}+R \nu^{\prime}\right)}
$$


which shows that if we choose $R=r \mu(t)$ then $\rho$ will be independent of $r$ i.e., $\rho^{\prime}\left(t, r, x_{1}, \ldots, x_{n-2}\right)=0$. It is to be noted that $\rho$ will also become independent of other space co-ordinates. Also the choice $R=r \mu(t)$ results $\sigma=0$. Hence the space-time is homogeneous and isotropic. In proposition II, we have shown that $\sigma=0$ results to a black hole as the final state of collapse. Hence the proof.

From the above propositions it is clear that if the space-time is homogeneous and isotropic then the final outcome of collapsing process will be definite (a black hole) but no such conclusion is possible if the space-time is inhomogeneous and anisotropic.

\section{B. Transformation of the initial data set}

In the initial data set $\mathcal{I}=\{F, f\}$ if we make a linear transformation [10]

$$
\mathcal{L}_{T}: \mathcal{I} \rightarrow\left\{a^{\frac{n-1}{2}} F, \text { af }\right\} \quad(a>0, \text { a constant })
$$

then the scale factor $R$ changes as $R \rightarrow a^{1 / 2} R$ while $(\rho, \sigma)$ are invariant under this transformation but not the initial density and shear i.e., $\left(\rho_{i}, \sigma_{i}\right)$. In other words, there are classes of values of $F$ and $f$ for which we have the same values of $\rho$ and $\sigma$ but the initial ones (namely $\rho_{i}$ and $\sigma_{i}$ ) are different for each set of $(F, f)$. So it may happen that for one initial data set $(F, f)$ we have naked singularity as the final end state but choosing ' $a$ ' appropriately we have the transformed $(F, f)$ for which we may black hole as the final outcome of the collapsing process but $\rho, \sigma$ remain same for both the initial data set. This has been shown numerically for spherical collapse by Mena et al [10]. So we may conclude that energy density and anisotropy scalar at any time instant can not uniquely characterize the nature of the final state of collapse. But for the initial nature of the space-time no such conclusion can be made. In fact for a given initial inhomogeneity and anisotropy the initial data set can be specified uniquely and hence final outcome of the collapsing process may be characterized.

\section{CONCLUSIONS}

In this paper, we have studied properties of anisotropy and inhomogeneity of the space-time both initially and at any epoch in the context of gravitational collapse. We have obtained two distinct features from these studies namely: (a) the matter density and shear scalar at any instant remain invariant under the linear transformation (29) and hence can not characterize the nature of the final singularity, (b) the initial energy density and shear scalar are not invariant under the linear transformation (29) but together can uniquely specify the initial data set and hence they may characterize the final outcome of the collapsing process. This is a very peculiar feature of the physical process because physical parameters

like energy density, shear must have consistent behaviour for all time, they should not have different characters initially and at any epoch. It seems that the trouble is with the initial choice of the scale factor. We shall discuss the role of initial choice in a subsequent paper.

\section{Acknowledgement:}

The authors are thankful to IUCAA for worm hospitality where the major part of the work has been done. One of the authors (U.D) thanks CSIR (Govt. of India) for the award of a Senior Research Fellowship.

\section{References:}

[1] S. W. Hawking and G. F. R. Ellis, The large scale structure of space-time (Cambridge. Univ. Press, Cambridge, England, 1973). 
[2] R. Penrose, Riv. Nuovo Cimento 1252 (1969); in General Relativity, an Einstein Centenary Volume, edited by S.W. Hawking and W. Israel (Camb. Univ. Press, Cambridge, 1979).

[3] For recent reviews, see, e.g. P. S. Joshi, Pramana 55529 (2000); C. Gundlach, Living Rev. Rel. 24 (1999); A. Krolak, Prog. Theo. Phys. Suppl. 13645 (1999); R. Penrose, in Black holes and relativistic stars, ed. R. M. Wald (Univ. of Chicago Press, 1998); T. P. Singh, $g r-q c / 9805066$.

[4] P. S. Joshi and I. H. Dwivedi, Commun. Math. Phys. 166117 (1994).

[5] P. S. Joshi and I. H. Dwivedi, Class. Quantum Grav. 1641 (1999).

[6] K. Lake, Phys. Rev. Lett. 683129 (1992).

[7] A. Ori and T. Piran, Phys. Rev. Lett. 592137 (1987).

[8] T. Harada, Phys. Rev. D 58104015 (1998).

[9] P. S. Joshi, Global Aspects in Gravitation and Cosmology, (Oxford Univ. Press, Oxford, 1993).

[10] F. C. Mena, B. C. Nolan and R. Tavakol, $g r-q c / 0405041$.

[11] P. S. Joshi and A. Krolak, Class. Quantum Grav. 133069 (1996).

[12] U. Debnath, S. Chakraborty and J. D. Barrow, Gen. Rel. Grav. 36231 (2004); U. Debnath and S. Chakraborty, JCAP 05001 (2004).

[13] P. Szekeres, Phys. Rev. D 122941 (1975); S. Chakraborty and U. Debnath, grqc/0304072; Int. J. Mod. Phys. D 131085 (2004).

[14] P.S.Joshi, N.Dadhich and R.Maartens, Phys. Rev. D 65 101501(R) (2002). 